\title{
DENGUE: BREAKBONE FEVER, HEMORRHAGIA OR SHOCK
}

\section{Shamala D}

Department of Medical Microbiology,Faculty of Medicine, University of Malaya, Kuala Lumpur.

\begin{abstract}
:
Dengue virus infection has been endemic in Malaysia and in the region for decades and the disease caused by the virus has led to increased morbidity and mortality each year. Dengue is now endemic in more than 110 countries worldwide with two-fifths of the world's population at risk. Dengue fever is a flu-like illness and clinical features vary with the age of the patient. Dengue haemorrhagic fever on the other hand, is characterized by high fever, haemorrhagic phenomena and in severe cases, circulatory failure. There is no specific treatment for dengue fever but careful clinical management frequently saves the lives of these patients. Vaccine development has been difficult as protection against one or two dengue viruses could actually increase the risk of more serious diseases. At present, the only method of preventing dengue is to combat the vector mosquitoes. Dengue is diagnosed by viral isolation and or serology. Current methods, some in-house and others commercial, are far from satisfactory. My laboratory has developed, evaluated and validated tests for dengue that now enable detection on the first day of illness in most cases. At the same time an important aspect of dengue prevention is protection. To this end, we have been investigating the various immune parameters such as T-cells, cytokines and antibody neutralizing activities to show that specific immune responses do occur in a selected Malaysian population which are HLA restricted, cytokine mediated and directed specifically at certain regions of the virus. (JUMMEC 2008; 11 (2): 39-52)
\end{abstract}

KEYWORDS: dengue, dengue haemorrhagic fever, laboratory diagnosis

\section{Introduction and Epidemiology}

Dengue and dengue hemorrhagic fever (DHF) are caused by one of four closely related, but antigenically distinct virus serotypes (DEN-1, DEN-2, DEN-3, and DEN-4) of the genus Flavivirus (1). Infection with one of these serotypes does not cross-protect, so persons living in a dengue-endemic area can have up to four dengue infections during their lifetime. Dengue is an urban disease of the tropics and the viruses that cause it are maintained in a cycle that involves humans and Aedes aegypti; a domestic, day-biting mosquito that prefers to feed on humans. In some regions, other Aedes species such as Ae. albopictus and Ae. polynesiensis are also involved. Infection with a dengue virus can produce a spectrum of clinical illness which ranges from a non-specific viral syndrome to a severe and fatal hemorrhagic disease. Important risk factors for DHF include the strain and serotype of the virus, age, immune status and genetic predisposition of the patients $(2,3)$.

Dengue is the most important arthropod-borne viral disease of humans worldwide with an estimated 100 million cases of dengue fever (DF) and 250,000-500,000 cases of DHF in the world, with the average case fatality rate being $5 \%$. Half of the world's population lives in areas at risk of infection and these are popular tourist destinations, too. For the first 5 years of the current decade, the annual average number of cases was 925,896, almost double the figure for 1990-1999 $(479,848$ cases) (Figure 1). All four dengue viruses are now circulating in Asia, Africa and the Americas. It is a scene that did not prevail two or three decades ago. Perhaps the only comforting news is that reported casefatality rates have been lower in recent years than in the decades before 2000 (4). The earliest record of dengue in Malaysia was published by Skae in 1902 of an outbreak in Penang (5). Subsequently in the years 1904, 1932 and

Correspondence:

Shamala Devi

Department of Medical Microbiology

Faculty of Medicine

University of Malaya

50603 Kuala Lumpur

Email: shamala@ummc.um.edu.my 


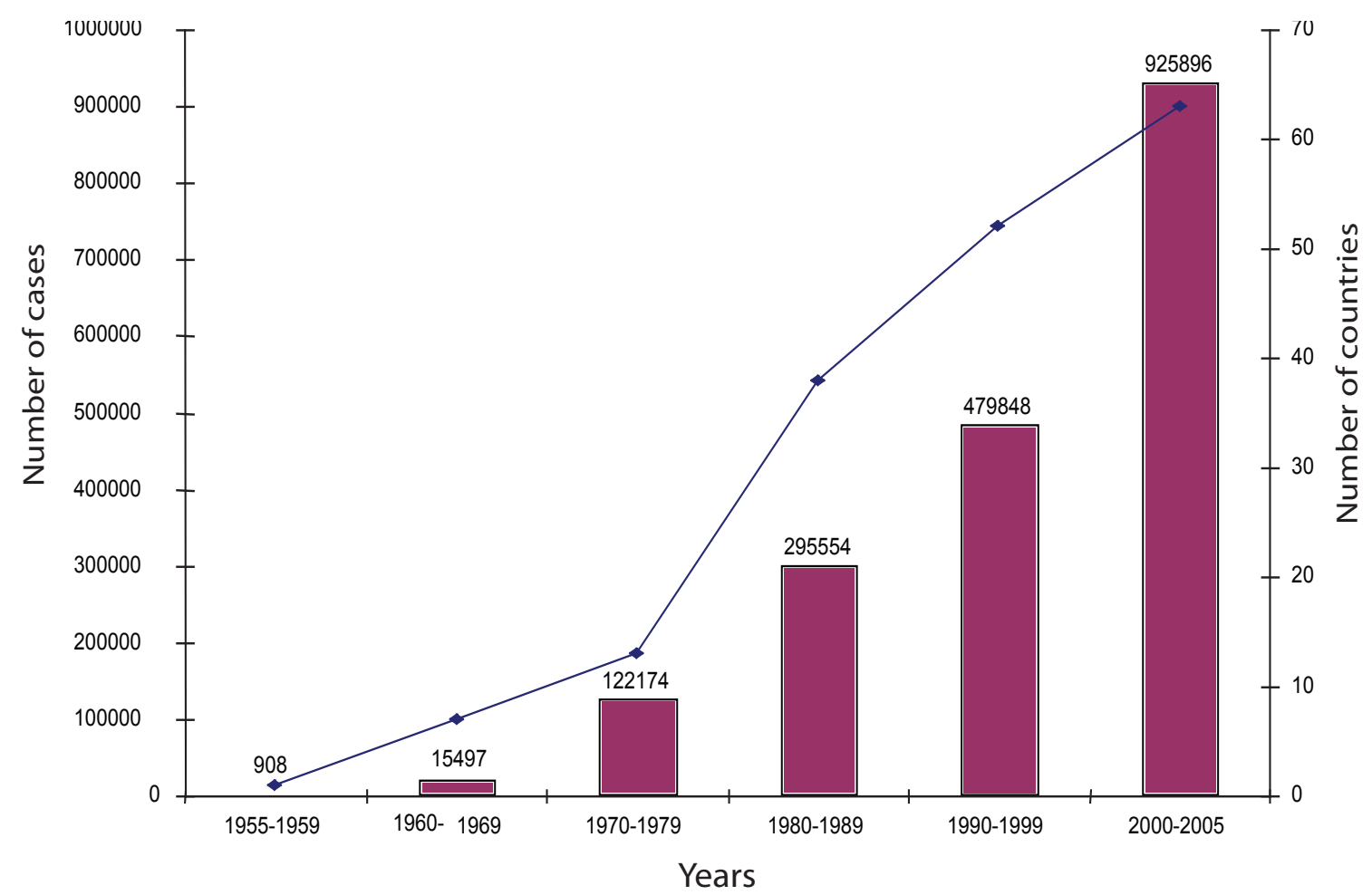

Figure 1: Average number of cases of dengue or severe dengue reported to $\mathrm{WHO}$, and average annual number of countries reporting dengue from 1955 to 2005

1933, more reports were made of dengue outbreaks in various parts of Peninsular Malaysia, largely in ports and large cities (6). Dengue virus was first isolated in 1950 by Smith (7) and the first laboratory confirmation was carried out by the Institute of Medical Research in 1953. Since 1953, pockets of epidemics have occured in urban areas of Peninsular Malaysia. In Malaysia, dengue was made a notifiable disease in April 1971 (8). From then on, increased numbers were noted and dengue fever, dengue hemorrhagic and dengue shock syndrome DF/DHF/DSS have became major public health problems. Between 1973 and 1987, the mean incidence of DF was noted to be 2-11 per 100,000 while that of DHF was $0.5-8$ per 100,000 . The major ethnic group affected were the Chinese with the major age group being 10-19 years. A seasonal pattern was noted with cases increasing following the rainy seasons. Most cases occurred in urban areas. Reasons cited for the various outbreaks, as well as for the increase in cases, were: the exodus of susceptibles from non-endemic to endemic areas, rapid urbanization, a disturbance in human ecology, creation of slums and squatters and living conditions that perpetuated vector breeding $(9,10)$. Figure 2 depicts the difference in clinical versus serologically confirmed dengue cases from 1973 (11). As can be seen, the number of cases confirmed by serology is 11,930 as compared to the number of cases reported which is 44,189 (as of November 2007-www.moh. gov.my). The DHF case fatality rate is $3.6 \%$ while that for total dengue is $0.26 \%$.

The reasons for this dramatic global emergence of dengue/DHF as a major public health problem are complex and not well understood. However, several important factors have been identified: ineffective mosquito control, which is virtually nonexistent in most dengue-endemic countries, and global demographic changes such as uncontrolled urbanization and concurrent population growth resulting in substandard housing and inadequate water, sewer, and waste management systems. All of these increase Ae. aegypti population densities, thus facilitating transmission of Ae. aegypti-borne disease (12). Consumer goods packaged in non-biodegradable plastic and automobile tyres, discarded into the environment, provide ideal larval habitats that lead to increased population densities of the principal mosquito vector, Ae. aegypti. Finally, increased travel by airplane has helped transport dengue viruses between population centers of the tropics, resulting in a constant exchange of dengue viruses and other pathogens (13). 


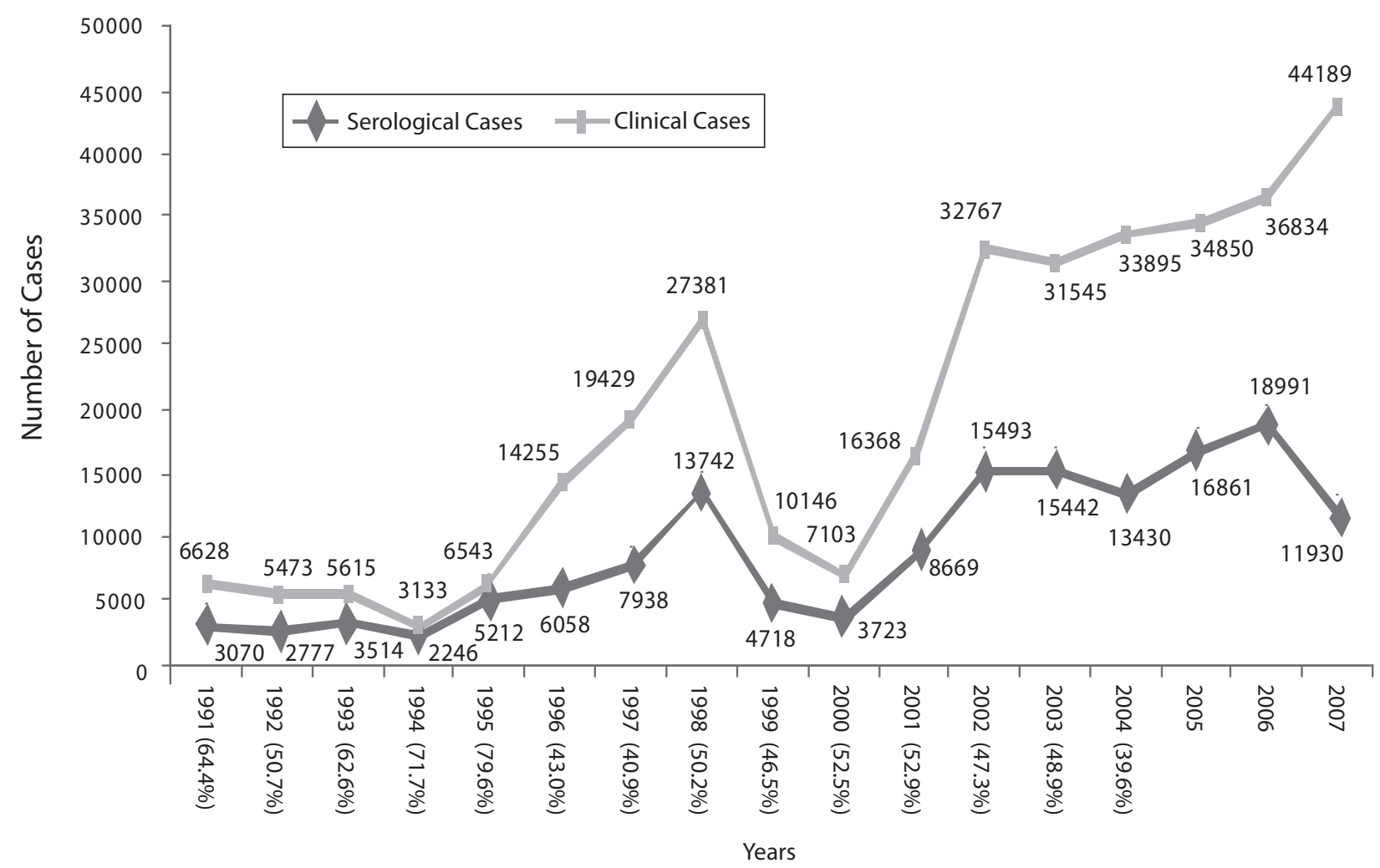

Figure 2: Distribution of Clinical and Serologically Positive Cases of Dengue in Malaysia: Source: Vector Borne Disese Division, $\mathrm{MOH}$

\section{Clinical Disease}

DF may be asymptomatic or lead to a range of clinical presentations even death. Clinically, it can be confused with influenza, rubella, malaria, chikungunya, leptospirosis or typhoid. The incubation period is four to seven days (range of 3-14). The inoculation of the dengue virus to the human host starts with the bite of the infected female mosquito vector that introduces saliva containing the dengue virus into the bite site which then spreads to the possible target tissues such as lymph nodes, spleen, bone marrow and liver. The incubation period ends with an acute onset of fever, which might recur after five to eight days. During the febrile phase of the viral infection, viremia occurs and lasts for three to five days. Together with the occurrence of viremia and fever, prodromal syndromes such as malaise, chills, and headache may develop. These are followed by muscle, back, retro-orbital and joint pain. The febrile painful period of DF lasts five to seven days, and may leave the patient feeling tired for several more days. The vast majority of infections are asymptomatic or minimally symptomatic. Leukopenia and mild thrombocytopenia are frequently seen as well as hemorrhagic manifestations such as petechial rash, epistaxis, gum bleeding, gastrointestinal bleeding, microscopic hematuria and hypermenorrhea. The more severe manisfestation is DHF. Once primarily a children's disease, it is now seen in all age groups $(14,15,16)$. Most cases occur in individuals with prior dengue exposure. DHF has been classified into 4 grades according to severity of shock and bleeding. It is defined as an acute febrile illness, with minor or major bleeding, thrombocytopenia, plasma leakage, pleural or other effusions or hypoalbuminemia/proteinemia. Pathophysiologically, plasma leakage differentiates DF and DHF. A positive tourniquet, collection of exudates at pleural and abdominal cavities, a progressively decreasing platelet count and a rising hematocrit (signifying abnormal capillary permeability) indicate increased probability of impending shock (17). Once shock has set in, the fatality rate may be as high as $12-44 \%$. Liver damage, cardiomyopathy, encephalopathy, neurological manifestations such as altered consciousness, convulsions and coma have also been described (18). The definition of severe dengue is currently under review. An immunological basis for endothelial permeability and vascular leakage is widely accepted, but definitive data to explain the mechanism is still lacking (19). 

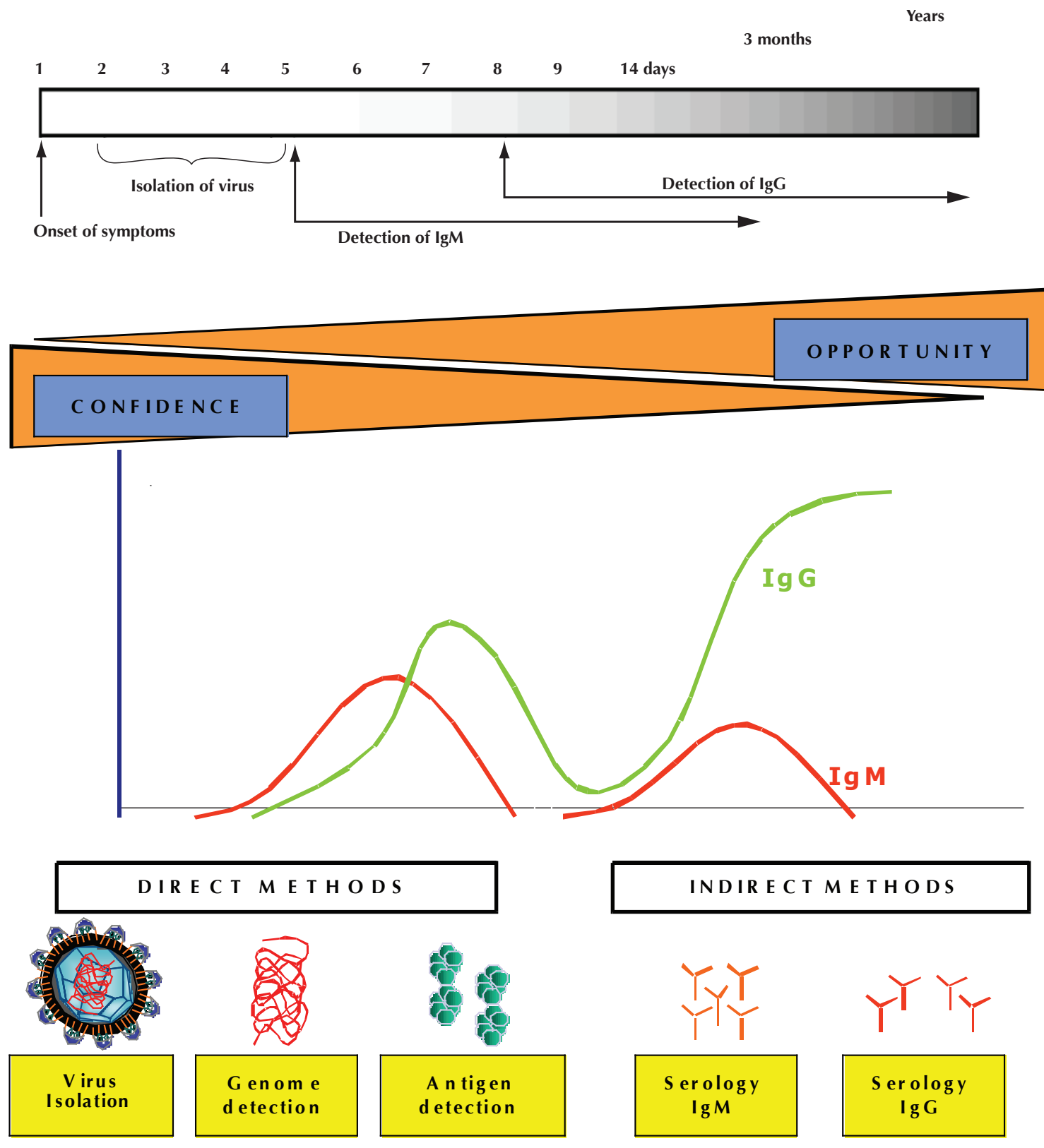

Figure 3: Suitability of dengue diagnostic assays during the course of the disease

\section{Laboratory Diagnosis}

Dengue viruses belong to the family Flaviviridae that has more than 70 viruses that cross-react in serological tests as they share group antigens, thus complicating diagnosis. Laboratory diagnosis depends on virus isolation and serologic tests (20). Circulating virus remains detectable in the blood during the febrile period after which they are rapidly cleared with the appearance of specific antibody. Virus isolation is carried out using mosquito cell lines which is then detected using an indirect fluorescent antibody test. The use of polymerase chain reaction (PCR) may shorten the time of detection (21), but this test is still experimental and the few commercial products available have not been validated. Serological diagnosis depends on the presence of IgM antibody or a rise in IgG antibody titer in paired acute and convalescent phase sera. More than $90 \%$ of patients are $\lg \mathrm{M}$ positive by the fourth day of illness, but the IgM antibody may be due to an infection up to 3 months earlier $(22,23)$. Commercial kits for the measurement of antibodies include the ELISA kits, a dipstick and a rapid dot-blot assays. These kits do not require specialized training, but their sensitivity and specificity vary. The choice of a test therefore, 
depends on the availability of facilities, human resources and also time of sampling. Figure 3 shows the comparison of tests according to the assessibility of the tests and their confidence. However for a diagnosis of "confirmed" dengue, the dengue virus should be identified by isolation or there could be a four-fold rise in antibody titer. Recent innovations in the development of dengue diagnostics include NS1 ELISAs (24), chemiluminescent optical biosensors for the detection of virions, natural cytotoxicity receptor immunoglobulins, recombinant proteins, microsphere based immunoassays and lab on a CD assays (25).

\section{Pathogenesis}

The risk of DHF is higher when two or more virus isotypes are circulating simultaneously. Also the presence of dengue antibodies acquired either actively by prior infection or passively via maternal antibodies in milk or in utero is a contributory factor. Thus, antibody actually enhances viral infectivity at non-neutralizing concentrations. During the primary infection, dengue virus attaches to the target cell via a highly sulphated glycosaminoglycan heparan sulfate. This is followed by the penetration of virus into the target cell, mediated by another secondary high affinity receptor. The identity of the high affinity receptor in dengue virus infection has yet to be resolved and further studies are required as the list of proposed and known receptors including DCSIGN and other unidentified proteins are tremendous. In secondary infection of dengue virus, it is believed that apart from the typical infection route as demonstrated in the primary infection, dengue virus mediates its entry into target cells via $\mathrm{Fc}_{\mathrm{Y}}$-receptors. The hypothesis known as antibody-dependant enhancement (ADE) is a process in which the virus complexed with specific antibody is taken up by mononuclear cells via a FcR mediated endocytosis (19). This mode of entry takes advantage of the host's immune system and has led to the extensive infection of the cells possessing $\mathrm{Fc}_{\gamma} \mathrm{R}$ such as monocytes, dendritic cells and B-lymphocytes. The infection of these cells results in release of pro-inflammatory chemokines that are implicated in the development of plasma leakages in DHF and dengue shock syndrome (DSS). Chemical mediators such as interleukin-1, interleukin-6, tumor necrosis factor and platelet activating factor from infected monocytes, gamma interferon, interleukin-2, interleukin-4, interleukin-5, interleukin-6, and interleukin-10 produced by Tlymphocytes are suspected of playing essential roles in the vascular endothelial damage of the disease, the development of severe plasma leakage, and the hemorrhagic manifestations. But this hypothesis is not unanimous as there is a small but consistent percentage of DHF/DSS cases that are due to primary infections (26), that is, no pre-existing antibody in these individuals. Epidemiological and laboratory studies suggest also that virus strain, individual susceptibility and good nutritional status may also be important as risk factors for DHF. Therefore to ensure that a vaccine gives immunity and does not enhance immunopathology, it must give protection to all the four serotypes, give high levels of immunity and give life-long immunity. Many approaches are being taken in vaccine development such as recombinant subunit vaccine, live recombinant vaccines and live attenuated vaccines. The lack of understanding of the immunology and immunopathology of the disease and of a suitable animal model as well as the inherent dangers of using live vaccines, have made vaccine development slow. Currently, candidate attenuated vaccines are being evaluated in various parts of the world.

\section{Prevention and Control of Dengue Infections}

Prevention and control currently depends on controlling the mosquito vector, Ae. aegypt, in and around the home where most transmission occurs. Space sprays with insecticides to kill adult mosquitoes are not usually effective as ULV-aerosols have little impact on the adult female Ae. aegypti and no impact on the immature stages. The most effective way is larval source reduction that is, eliminating or cleaning waterholding containers that serve as the larval habitats in the domestic environment. The feeding habit of Ae. aegypti, which involves a typical pattern of high frequency-short period blood meals taken from several victims in every meal, has increased the odds for the dengue virus to spread with high efficacy. Moreover, Ae. aegypti prefers to live and feed in indoor domestic environment. The period for the infected mosquito to become infectious is approximately eight to twelve days after an infected blood meal. In addition to the infected female mosquito, transovarial transmission has been demonstrated in various studies (27). Studies have shown that $2.35 \%$ to $40.00 \%$ of mosquito larvae were found to be positive for dengue virus. The task might seem a simple matter of treatment and elimination of 
infested containers but they are hard to sustain as they are labour intensive, requiring discipline, diligence and are intrusive. In recent years, there has been an increased focus in the role of the community in mosquito control (13). Community participation requires extensive social marketing of dengue prevention, with health education and community outreach. Government participation in the form of elimination of mosquito production sites on a larger scale and some through limited use of larvicides and adulticides are essential. In addition, active laboratory based surveillance that can provide early warning for epidemic activity is essential. Health officials should know at any point in time where dengue transmission is occurring, what serotypes are circulating and the severity of illness associated with dengue infection. Another equally important component of a sustainable prevention programme is the education of the medical community on clinical diagnosis and management of DHF cases so as to help understand the pathophysiological changes that occur in DHF and hence, keep fatalities low. Furthermore, greater research efforts looking into more effective disease prevention strategies such as new mosquito control technology, the changing epidemiology, disease pathogenesis and dengue vaccines are essential. Lastly, community-integrated programmes would certainly be useful not only for keeping dengue epidemics at bay but also all other infectious diseases transmitted similarly and with an endemic nature.

\section{Dengue Vaccines and Antivirals}

To date, no vaccine has yet been licensed and no candidate vaccine has progressed beyond phase II clinical trials. It generally takes 15 years for candidate vaccines to advance from discovery to licensing. The presence of four serotypes, the problem of immune enhancement and the complexity of the disease are some of the factors that have impeded the development of dengue vaccine. The theoretical danger of a dengue vaccine that causes a severe disease exists unless solid immunity is afforded equally to all the serotypes. Another point to note is that the correlates of protection to dengue are poorly understood and hence, intensive studies are essential to demonstrate that any candidate vaccine induces only a protective response.

To add to this problem, the lack of a suitable animal model has hampered assessment of any developing vaccines. As such, clinical trials are important in terms of the information they provide on immunity and reactogenicity while long term evaluation is required to demonstrate lack of evidence of immune enhancement. Currently a number of vaccines are in clinical trials (Table 1): live attenuated tetravalent

Table I: Types of Dengue vaccines being developed and their current status; Adapted from reference 55

\begin{tabular}{|c|c|c|c|}
\hline Type of Vaccine & Key Features & Institute & Current Status \\
\hline \multirow[t]{2}{*}{ Live Attenuated } & $\begin{array}{c}\text { Serial passage of DEN } 1,2 \& 4 \text { in } \\
\text { PDK* cells }^{*} \text { \& }\end{array}$ & Mahidol University & Phase 2 Clinical Trials \\
\hline & $\begin{array}{l}\text { DEN-3 in vero cells and final passage } \\
\text { in FRhL\# }\end{array}$ & Aventis Pasteur, France & \\
\hline Live Attenuated & DEN 1-4 Serial passage in PDK cells & $\begin{array}{l}\text { Walter Reed Institute/Glaxo } \\
\text { SmithKline, Belgium }\end{array}$ & $\begin{array}{l}\text { Phase } 2 \text { Clinical } \\
\text { Trials-Children }\end{array}$ \\
\hline Chimeric & $\begin{array}{c}\text { DEN 1-4 prM and E genes inserted } \\
\text { into non structural portion of } \mathrm{YF}+ \\
17 \mathrm{D} \text { vaccine }\end{array}$ & $\begin{array}{l}\text { St. Louis University } \\
\text { Acambis Cambridge } \\
\text { Aventis Pasteur, France }\end{array}$ & Phase 2 Clinical Trials \\
\hline Chimeric & $\begin{array}{l}\text { DEN } 1-3 \text { prM and E genes inserted } \\
\text { into attenuated DEN-4 backbone }\end{array}$ & $\mathrm{NIH}$, USA & Volunteer Trials \\
\hline Chimeric & $\begin{array}{l}\text { DEN 2-4 prM and E genes inserted } \\
\text { into attenuated DEN-1 backbone }\end{array}$ & NIH, USA & Phase 1 Trials \\
\hline Chimeric & $\begin{array}{c}\text { DEN } 1 \text {-4 prM and E genes inserted } \\
\text { into attenuated DEN-2 }\end{array}$ & CDC, USA & In vivo (mouse) \\
\hline
\end{tabular}

*PDK - primary dog kidney cell line 


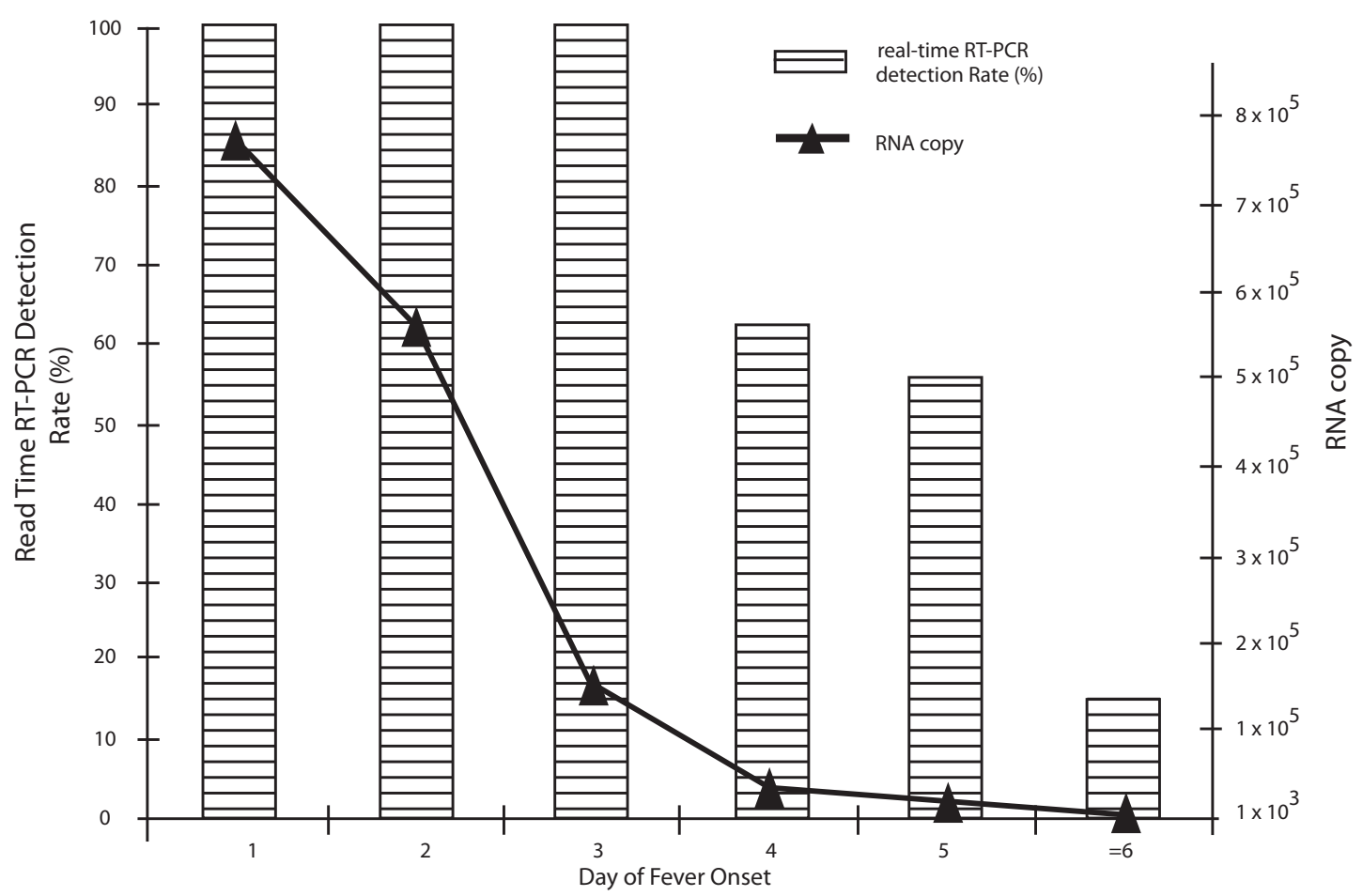

Figure 4 : Comparison between viral detection rate, day of fever onset and RNA copies

candidates utilizing classical virological techniques which are in phase II trials and recombinant vaccines (Chimerivax) that use the 17DYF vaccine as a backbone, which are also in phase II clinical trials (28). Candidate recombinants using DEN-4 as the backbone are also in phase I and II clinical trials. Alternative approaches such as DNA vaccines have demonstrated immunity in non-human primates and are now in phase I clinical trials. The most advance subunit vaccine is based on the recombinant E and NS1 produced in a Drosophila expression system. This, however, is currently being tested in non-human primates. A major challenge exists in that the criteria that are to be used to evaluate candidate vaccines in exposed populations as the definition of protection to dengue are not yet scientifically demonstrated. On a related note, there have also been developments in antiviral drugs and similar constraints do exist. There have been some exciting developments and drugs have been designed specifically targetting the NS3 and NS5 protein (29). Apart from these, natural products from plants have also been shown to be effective, less cytotoxic and are said to be cheaper (30).

\section{Research}

My advent with dengue virus began with the demonstration of a cellular immune response to dengue in mice and the presence of $\mathrm{H}-2$ restricted cytotoxic activity to dengue virus infections in mice (1982-1986 - PhD thesis, 31, 32, 33, 34, 35). Following that, I then went on to develop monoclonal antibodies to dengue virus (1987-1991, as a co-researcher), studied the epidemiology of dengue in Malaysia (1985-1990, 36, 37), and also investigated possible role of cytokines in dengue infections (1996-1997, 38). To address some of the questions raised above, my laboratory embarked on determining what proteins are produced in response to this virus both in the cells and in the plasma. This is with the aim of identifying early and newer biomarkers that could then be potential diagnostic reagents. Diagnostic tools were developed such as Real Time PCR assays and current tools, such as commercial serological kits and antigen assays, were evaluated. I also hoped to develop a point of care test with reasonable sensitivity and specificity for use in the future. At the same time, I also began investigations into the mechanisms of protection and began investigating HLA associations and the cellular immune response specifically to the $T$ cells and attempted to determine the viral epitopes selected by the host in its development of specific $T$ cells as these are essential correlates of immunity for any intracellular organism. These studies would also have an impact on vaccine assessment and development. To further address newer syndromes and 

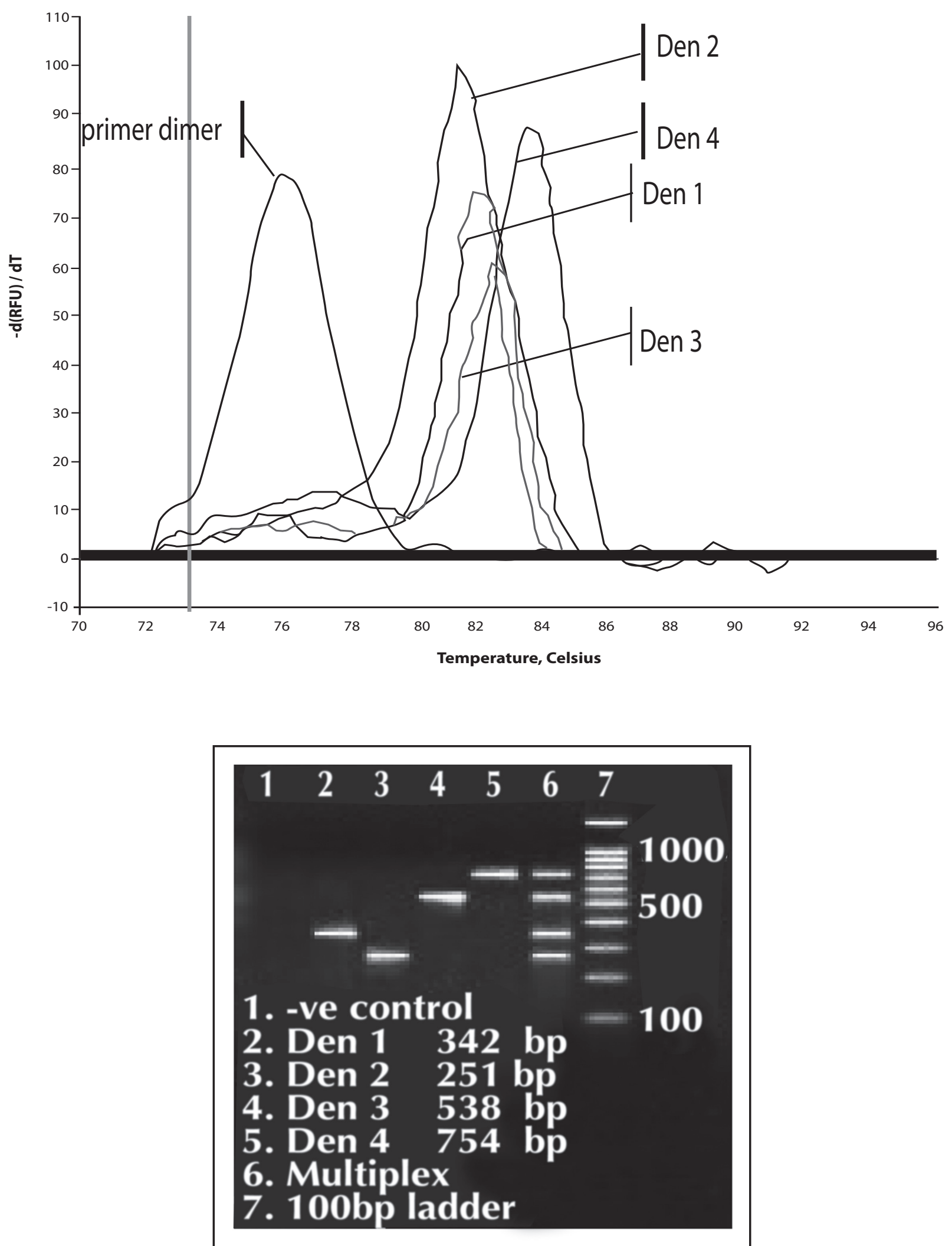

Figure 5: Multiplex RT-PCR analysis by Standard $P C R$ and Real Time PCR 
possible changes in the virus itself, sequencing of the viruses isolated in the region was carried out from time to time.

\section{Development of Molecular Diagnostic Tests}

The genome of the dengue virus encodes for three structural proteins ( $C$, preM and $E$ ) and seven nonstructural proteins (NS1, NS2a, NS2b, NS3, NS4a, NS4b and NS5). Among the structural proteins, the E protein serves as a hemagglutinin and surface molecule. It possesses the strain determinant of the virus and is the main target of the humoral immune response. The non-structural protein 1 (NS1) is said to serve as a viral genome replicating factor associated with the negative strand of the viral RNA and it is also secreted. The function of the hydrophobic NS2a, NS4a and NS4b are still obscure, but they have been postulated to serve as anchors for viral replication complex or to inhibit the interferon response of host's cell (NS4b). NS3 together with NS2b form a serine-protease to cleave the precursor protein formed in the primary translation process. Finally, the NS5 protein serves as the RNA-dependent RNA polymeraseand methyltransferase in the replication of the virus (39). With this knowledge two multiplex PCRs (Standard and Real Time) were developed to two different regions of the virus (preM-Capsid and NS5) in order to further improve diagnostics of dengue infections. Both assays have shown $100 \%$ specificity and are more than $98 \%$ sensitive, and they are able to detect/amplify virus from Day 1 of onset of fever (Figure 4 and 5). Viral antigens can be detected up to Day 7 of fever and even in the presence of high titres of circulating
Table 2: Sensitivity, Specificity and Efficiency of NS1 ELISAs as compared to RT-PCR

\begin{tabular}{lccc}
\hline Assay & Sensitivity (\%) & Specificity (\%) & Efficiency (\%) \\
\hline RT-PCR & 97 & 100 & 98 \\
Biorad NS1 & 87 & 100 & 89 \\
Panbio NS1 & 84 & 100 & 73 \\
\hline
\end{tabular}

antibodies $(40,41)$. The laboratory has also evaluated two commercially available ELISA kits for the detection of NS1 and the results (Table 2) imply that it is a useful assay in the first four days of fever, but its detection rate decreases with increasing levels of antibody (24). To enhance diagnostics for dengue virus infections, we utilized two-demonsional electrophoresis, mass spectrometry and Western blot to determine the differential expression levels of proteins in peripheral blood mononuclear cells (PBMC) of patients with DF and DHF in order to identify both viral factors and host factors that may be used as either diagnostic reagents or as early diagnostic markers for disease severity. Eight proteins were identified that were up-regulated two-fold or more in patients compared to healthy controls of which at least three proteins-aldolase, thioredoxin peroxidase and alpha tubulin-are related to dengue infection. Both thioredoxin peroxidase and alpha tubulin are over-expressed in DHF patients compared to DF patients while aldolase was expressed at a higher level in DF patients as seen in Table 3 (42, 43). We are currently developing methods that will be able to detect both antigens and antibodies simultaneously so that the disease can then be diagnosed irregardless of the day of onset of fever.

Table 3: MALDI-TOF identification of differentially expressed proteins in DF and DHF patients

\begin{tabular}{|c|c|c|c|c|c|}
\hline Proteins ID & Experimental MW/pl & Sequence Coverage & DF (\% Vol) & DHF (\%Vol) & $\begin{array}{l}\text { Image Master 2-D Elite } \\
\text { Software Analysis }\end{array}$ \\
\hline Alpha 1 Antitrypsin & $44.2 \mathrm{kD} / 5.4$ & 16.2 & 0.357 & 0.647 & 2 fold in DHF vs DF \\
\hline Dengue NS3 & 79 kD/4.5 & 15.7 & 0.235 & 0.502 & 2 fold in DHF vs DF \\
\hline Dengue NS1 & 50 kD/6.5 & 17.3 & 0.367 & 0.698 & 2 fold in DHF vs DF \\
\hline Aldolase & 39 kD/8.7 & 25.3 & 0.461 & 0.234 & 2 fold in DF vs DHF \\
\hline Alpha tubulin & 48 kD/5.0 & 14.2 & 0.138 & 0.458 & 3 fold in DHF vs DF \\
\hline Thioredoxin & $22 \mathrm{kD} / 5.7$ & 23.2 & 0.288 & 1.428 & 6 fold in DHF vs DF \\
\hline
\end{tabular}




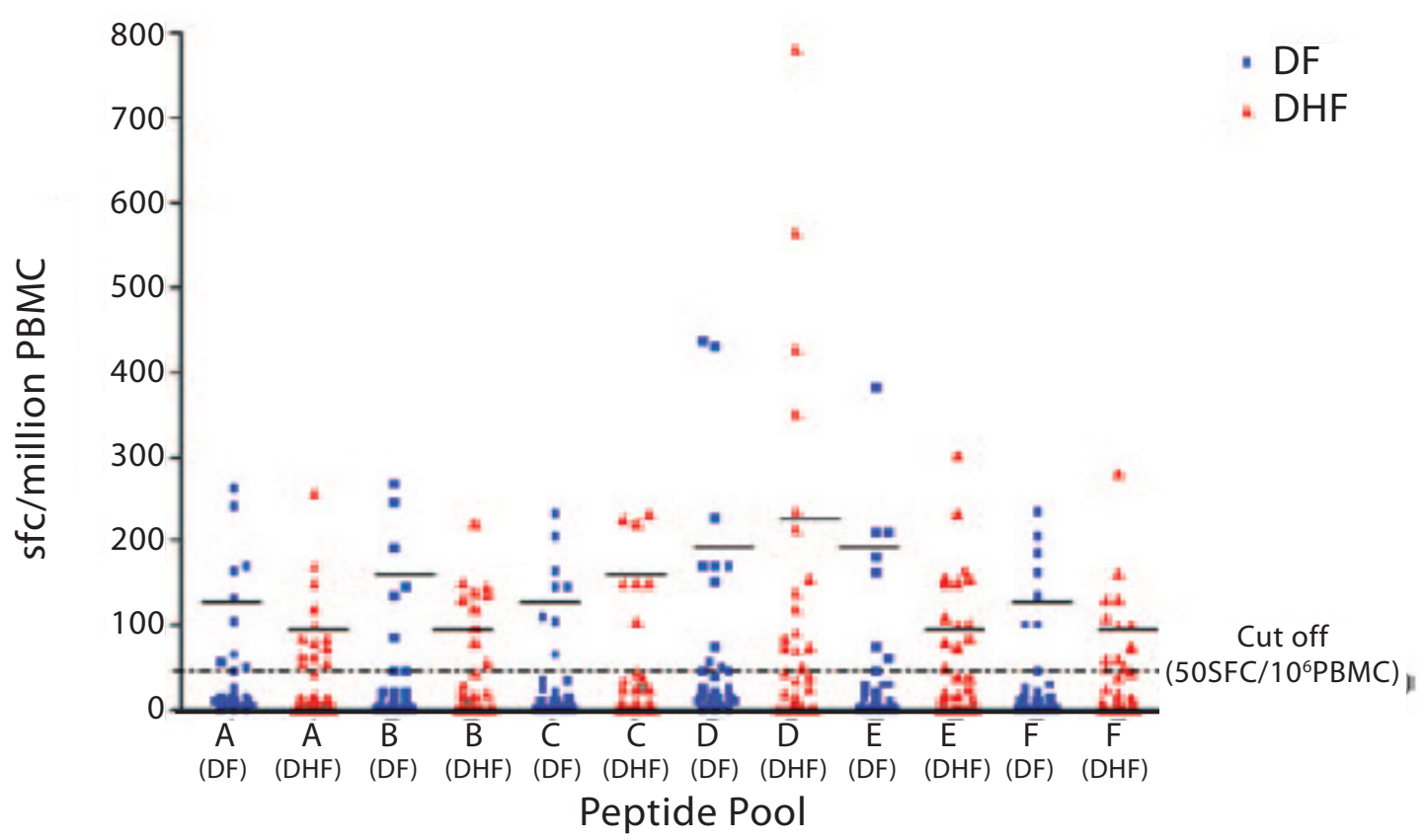

Figure 6: Spot-forming cells per million PBMC in DF versus DHF patients in response to peptide pools from various regions of the virus Levels of SFC in DF and DHF patients in response to peptide pools containing peptides from structural (AnC, C, PrM and E) and nonstructural (NS1, NS2A, NS2B, NS3, NS4A, NS4B, and NS5) regions. Pool A: AnC, C, PrM and E; Pool B: E, and NS1; Pool C: NS1, NS2A; Pool D: NS2B and NS3; Pool E: NS3, NS4A and NS4B; and Pool F: NS5. The means for each peptide pool is indicated by a bar. Responses were considered significant when a minimum of 50 SFCs per 106 PBMC were present per well, representing at least twice the number of SFCs in negative controls

\section{T Cell Responses in Dengue Fever and Dengue Hemorrhagic Fever Patients in Malaysia}

The magnitude of $\mathrm{T}$ cell responses and the involvement of cytokines and chemokines have been reported to correlate with dengue disease severity. In our study, 60 Malaysian adults with dengue viral infections were investigated for their dengue virus-specific $T$ cell responses to 32 peptides antigens from the structural and non-structural regions from dengue virus isolate. Seventeen different individual peptides from $C, E$, NS2B, NS3, NS4A, NS4B and NS5 were found to evoke positive gamma interferon responses by using an in-house developed enzyme-linked immunospot (ELISPOT) assay in 13 patients with DF and DHF with the range of $50-700 \mathrm{SFU} / 10^{6}$ PBMC. NS3 and predominantly $\mathrm{NS}_{422-431}$ peptide were found to be important T-cell targets (Figure 6, 44). The ELISPOT analysis also revealed high frequencies of $T$ cells that recognize both serotype-specific and cross-reactive dengue virus antigens in patients with DHF. The results strongly support the presence of high frequencies of activated $\mathrm{CD}^{+} \mathrm{T}$ cell in patients with $\mathrm{DHF}$, with the highest reactivity being targeted to NS3 region. These findings indicate a need to identify as many denguespecific T-cell peptides as possible in a larger number of dengue patients with multiple HLA backgrounds to better understand how the immune system responds tdengue virus and contributes to pathogenesis. Preliminary data on the level of cytokines indicate a probable role for IP-10, PDGF and IL-9 as these cytokines were elevated in DHF as compared to DF patients. Currently, an attempt is being made to further define the role of pro-inflammatory cytokines in vascular leakage and to determine that which leads to endothelial leakage.

\section{HLA-A and -B allele associations with DF and DHF patients in Malaysia.}

It has been proposed that HLA alleles play a significant role in the severity of dengue viral infections. The higher frequency of several HLA alleles in dengue infected patients versus control subjects would suggest that these alleles are associated with susceptibility or protection against this viral infection. In this study, we have investigated genotype variants of HLA Class 1 (-A and -B) of 50 dengue infected Malay, Chinese and Indian patients in Malaysia using polymerase chain reaction-sequence specific (PCR-SSP) techniques. The frequency of alleles $A^{*} 24$, $B^{*} 07$ and $B^{*} 40$ were significantly increased in dengue infected patients $(P C<.05)$ regardless of ethnicity, suggesting that patients with these alleles might be at increased risk to dengue viral infection. Significantly higher frequency of allele $B^{*} 13$ in dengue infected 
Malay patients suggests a probable role in susceptibility of dengue infection in this group. This finding on diverse genetic polymorphisms could serve as a prognostic tool to identify ethnically distinct individuals with risk for either disease severity and/or protection from severe disease. Further investigations are being conducted to show if this association is with a specific subtype.

\section{Sequencing of dengue viruses isolated in the region}

Preliminary study on dengue seroepidemiology in Brunei showed that from 2005 to 2006, dengue 2 was the predominant serotype followed by dengue 1 . This study is the first to report the isolation and typing of dengue viruses isolated in Brunei and the extent of dengue infection in that country (45). Limited sequence analysis of the E/NS1 gene at both amino acid and nucleotide level revealed that the Brunei DEN2 were mainly clustered in the genotype I together with the Malaysian, Indonesian, Sri Lankan and Myanmar isolates (46). Five genotypes were identified and the phylogenetic analysis of the full-length sequences places these DEN-2 isolates very near to the Indonesian and Australian strains. Nucleotide sequences showed changes occurred at 51 positions (21.2\%) while the amino acid homology ranged from $91 \%$ to $100 \%$ (47). Full sequence of two isolates were recently completed. A hundred Malaysian dengue viruses (1-4) isolated from the years 1995-2005 were sequenced and compared. This work is currently ongoing.

\section{Dengue specific receptors}

It is still unclear what receptors the dengue virus recognizes on the cell surface. Many cell types express heparan sulfate, but a more specific protein receptor is thought to be required to target dengue virus to permissive cell types. To date, glycans, DC-SIGN, GAGs, CD-14 associated protein and many other potential proteins have been suggested, but not confirmed, as possible modes of entry for this virus $(48,49)$. The primary viral receptor and the in vivo target cell needs to be determined unambiguously. With the advent of a modern day tool like RNA interference (RNAi), it is possible to home in on this elusive protein(s). RNAi is an evolutionarily conserved mechanism that permits the selective post-transcriptional down regulation of target genes in eukaryotic cells. As such, RNAi has enormous potential not only as an invaluable tool in biological research and drug development but also as a possible approach to the in vivo inactivation of gene products. We have designed siRNAs to silence potential targets in human cells to investigate if a putative receptor exists or if this virus utilizes something else for entry.

\section{Protection against dengue - what determines this?}

With the knowledge that pre-existing antibodies put us at risk of severe dengue infections, the question then arises as to what constitutes protection against dengue. A very large number of subclinical dengue infections are said to have occurred and this group is not accounted for in annual figures of dengue infections. These individuals have innate mechanisms of immunity that protect them. With this in mind, we have begun, firstly, to develop and establish assays that enable rapid detection of these antibodies using flow cytometry. This tool is being used to not only determine levels of neutralizing antibodies, but also to titrate the virus within 48 hours, a procedure that normally takes between seven to ten days. At the same time, the genetics of protection in this subclinically infected group will be determined. Another correlate of protection is the production of siRNAs by the host. To this end, the quatification of siRNAs to the virus is being attempted to determine the possibility of this tool in alleviating disease pathology. However, it is also imperative to monitor untoward off-target effects as well. This technology is also especially well-suited in treating viral infections, and numerous examples have now illustrated that a wide range of viruses can be inhibited with RNAi, both in vitro and in vivo.

\section{Primary Dengue Animal Model of human disease}

Dengue viral pathogenesis and vaccine studies are hampered by the lack of an ideal animal model mimicking human disease and eliciting an adaptive human immune response. Although currently available animal models have been very useful in dissecting some key aspects of disease pathogenesis, a major limitation with these models is the lack of a human immune response. In this study, we sought to overcome this difficulty by utilizing a novel mouse model that permits multi-lineage human hematopoiesis and immune responses following transplantation with human blood forming stem cells. To generate immunocompetent humanized mice, neonatal RAG2-/- $ү \mathrm{c}-/$ - mice were xenografted with human CD34+ hematopoietic stem 
cells, resulting in de novo development of major functional cells of the human adaptive immune system. To evaluate susceptibility to dengue viral infection, humanized mice were challenged with DEN-2 serotype. Viremia lasting up to three weeks was detected in infected mice with viral titers reaching up to $10^{6.3}$ RNA copies/ml. Fever characteristics of dengue were also noted in infected mice. The presence of human anti-dengue antibodies was evaluated using an antibody capture ELISA. Anti-dengue IgM was first detected by two weeks post-infection followed by IgG at six weeks. Sera from some of the infected mice were also found to be capable of dengue virus neutralization. Infected mouse sera showed reactivity with the viral envelop and capsid proteins in immunoprecipitation assays. These results (as summarized in Figure 7) demonstrate for the first time that humanized mice are capable of dengue viral primary human immune responses, thus paving the way for new dengue immunopathogenesis and vaccine studies (50). This study was carried out during my sabbatical at the Colorado State University in early 2006.

\section{Acknowledgements}

This study was funded by grants from the University of Malaya, the Academy of Sciences and the Ministry of Science and Technology, Malaysia. Sincere and grateful thanks to all my students and research assistants, both past and current, for their assistance in conducting the research for their postgraduate degrees; especially Dr Geetha Subramaniam, Ew Cheng Lan, Hooi Poh Sim, Leela Rajamani, Ramapraba Appanna, Ravindran Thayan, $\mathrm{Hj}$ Osmali Osman, Albert Lim and Mr Yong Yean Kong, Special thanks also to my clinical colleagues, Professor Lucy Lum, Dr Tan Lian Huat and their dedicated nurses and medical officers for the collection of samples without which this project would not been conducted; and my collaborators, Professor Ramesh Akkina, Jes Kuruvilla, Professor Rohana Yusuf, Dr Wouter Schul and Professor Martin Dorf.

\section{References}

1. Gubler DJ, Clark GG. Dengue/dengue hemorrhagic fever: The emergence of a global health problem. Emerging Infectious Diseases 1995; 1(2): 55-57.
2. Gubler DJ. The global pandemic of dengue/dengue haemorrhagic fever: current status and prospects for the future. Ann. Acad Med Singapore 1998; 27 : 227-234.

3. Rigau-Pérez JG, Gubler DJ , Clark GG, Reiter P, Sanders EJ, Vorndam AV. Dengue and dengue haemorrhagic fever surveillance. Lancet 1998; 352: 971-977.

4. World Health Organization fact Sheet No. 117. April 2002. Geneva.

5. Skae FMT. Dengue fever in Penang. Brit Med J 1902; 2:1581-1590.

6. Smith CGE. The history of dengue in tropical Asia and its probable relationship to the mosquito Aedes aegypti. J Trop Med Hyg 1956; 59:3-8.

7. Smith CEG. Isolation of three strains of type 1 dengue virus from a local outbreak of the disease in Malaysia. J of Hygiene 1956; 54: 569-580.

8. Wallace $H G$, Lim TH, Rudnick $A$, Knudsen $A B$, Cheong WM, Chew V. Dengue haemorrhagic fever in Malaysia: The 1973 epidemic. Southeast Asian J Trop Med Pub HIth 1980; 11:1-13.

9. Shekhar KC, Ong LH. Epdemiology of dengue/ dengue haemorrhagic fever in Malaysia - a retrospective epidemiological study, 1973-1987. Part II: Dengue Fever (DF). Asia Pacific J Pub HIth 1992; 6:126-133.

10. Shekhar KC, Senan P. Epdemiology of dengue/ dengue haemorrhagic fever in Malaysia III. A comparative study of clinical features seen in virologically confirmed cases for periods between 1963-1987 - a review. J Singapore Paediatric Soc 1992; 34: 67-82.

11. WHO Collaborating Centre for Arbovirus Reference and Research (DF/DHF) Malaysia. Annual report; 2006.

12. Leontsini E, Gil E, Kendall C, Clark GG. Effect of a community based Ae. Aegypti control programme on mosquito larval production sites in El Progreso, Honduras. Trans R Soc Trop. Med Hyg 1993; 87: 267271.

13. Gubler DJ, Clark GG. Community-based integrated control of Aedes aegypti: a brief overview of current programs. Am J Trop Med Hyg 1994:50:50-60. 
14. George R, et al. Changing pattern in the clinical presentation of dengue hemorrhagic fever in Malaysia during the period 1962-1982. J Malaysian Soc HIth 1984; 4:57-64.

15. George R. Dengue hemorrhagic fever in Malaysia (a review). South-east Asian J Trop Med Pub Hlth $1987 ; 18: 278-283$

16. Srichakul T, et al. Fibrinogen metabolism and the disseminated intravascular coagulation on dengue haemorrhagic fever. 1977; 26:525-532

17. Kliks SC, Nimmantiya S, Nisalak A, Burke DS. Evidence that maternal dengue antibodies are important in the development of dengue hemorrhagic fever in infants. Am J Trop Med Hyg 1988; 38(2): 411-419.

18. Lum LCS, Lam SK, George R, Devi S. Fulminant hepatitis and encephalopathy in dengue infection. South-east Asian J Trop Med Pub HIth 1993; 24:467471.

19. Halstead SB. Antibody, macrophages, dengue virus infection, shock and hemorrhage: A pathogenesis cascade. Rev Inf Dis 1989: 11: S830-839.

20. Dengue Haemorrhagic Fever: Diagnosis, treatment, prevention and control, 2nd ed. WHO, Geneva, Switzerland; 1977.

21. Brown JL, Wilkinson R, Davidson RN. Rapid diagnosis and determination of duration of viremia in dengue fever using polymerase chain reaction. Trans R Soc Trop Med Hyg 1996; 90: 140-43.

22. Lam SK, Devi S, Pang T. Detection of specific IgM to dengue infections. South-east Asian J Trop Med Pub Hlth 1987; 18 (14): 532-538.

23. Lam SK, Devi S. Further evaluation on the Dengue IgM ELISA. Arbovirus Information Exchange 1990; 203.

24. Devi S, Ew CL, Subramaniam G. Evaluation of a Dengue NS1 capture ELISA assay for the rapid detection of Dengue. J Infect Dev Countries 2007; 1(2):140-145.

25. Devi S, Artsorb H. Molecular diagnostics for the detection of flavivirus infections. Exp Opi Med Diag 2007; 1: 51-60.

26. Kuberski, Rosen L, Reed D, Mataika J. Clinical and laboratory observations on patients with primary and secondary dengue type 1 infection with hemorrhagic manifestations in Fiji. Am J Trop Med Hyg 1977; 26(4): 775-783.

27. Lee $\mathrm{HL}$, et. al. transovarial transmission of dengue virus in Aedes aegypti and Aedes albopictus in relation to dengue outbreak in an urban area in Malaysia. Dengue Bulletin 2005; 29: 106-111.

28. Edelman R. Dengue vaccines approach the finish line - review. Clin Infect Dis 2007; 45 (Suppl 1): S5660.

29. Schul W, Lin W, Xu HY, Flamond M, Vasudevan SG. A dengue fever viremia model in mice shows reduction in viral amplification and suppression of the inflammatory response after treatment with antiviral drugs. J of Infect Dis 2007; 195: 665-674.

30. Ono L, Wagner W, Iray MR, Terezinha LM, Coimbra, Philip AJG, Sierakowski MR. In vitro and in vivo antiviral properties of sulfated galactomannans against yellow fever virus (BEH111 strain) and dengue 1 virus (Hawaii strain). Antiviral Res 2003, 60: 201-208.

31. Pang T, Yap KL, Devi S, Wong PY. Delayed-type hepersensitivity (DTH) response to dengue virus infection in mice: Effect of route of sensitisation and splenectomy. Microbial Immunol 1983; 27(II): 945-951.

32. Pang T, Devi S, Wong PY, Mackenzie JFC, Yap KL. Ly phenotype and $\mathrm{H}-2$ compatibility requirements of effector cells in the delayed-type hypersensitivity response to dengue virus infection. Infect Immunol $1984 ; 4: 429-431$.

33. Wong PY, Devi S, Mackenzie JFC, Yap KL, Pang T. Induction and Ly phenotype of suppressor cells in mice during primary infection with dengue virus. Immunol 1984; 51: 51-56.

34. Devi S, Wong PY, Manickam L, Bomford R, Pang T, Dhaliwal SS. Effect of various adjuvants on the delayed-type hypersensitivity (DTH) response in experimental dengue infection. Trop Biomed 1985; 2: 6-11.

35. Pang T, Devi S, Blanden RV, Lam SK. T Cellmedicated cytotoxicity against Dengue-infected target cells. Microbiol Immunol 1988; 32(5): 511 518. 
36. Lam SK, Devi S, Pang T, Paul V, Teo L. Epidemiology of dengue in Malaysia in 1988. Dengue Newsletter 1990; 16: 35-40.

37. Lam SK, Devi S. Epidemiology of dengue in Malaysia. Dengue Newsletter, 16: 32.

38. Rajamani R, Nadia A, Devi S. Biological activities of dengue virus in mouse peritoneal cells. JUMMEC 1997; 2(1): 11-17.

39. Harris E, Holden KL, Edgil D, Polacek C, Clyde K. Molecular biology of flaviviruses. Novartis Found Symp 2006; 23-39.

40. Yong YK, Chong HT, Tan CT, Devi S. Rapid detection, serotyping and quantitation of dengue viruses by TaqMan Real-Time one-step RT-PCR. J Virol Methods 2006; 138: 123-130.

41. Yong YK, Thayan R, Chong HT, Tan CT, Devi S. Rapid detection and serotyping of dengue virus by Multiplex RT-PCR and Real-Time SYBR Green RTPCR. Sing Med Journal 2007; 48(7): 662-668.

42. Thayan R, Tan LH, Lum CS, Tan PL, Shahidah N, Rohana $Y$, Devi $S$. The use of two-dimension electrophoresis to identify serum biomarkers from patients with dengue haemorrhagic fever. In Trans Royal Soc Trop Med Hygiene; 2008.

43. Thayan R, Tan LH, Lum CS, Norshahidah K, Rohana $Y$, Devi S. Differential expression of aldolase, alpha tubulin and thioredoxin peroxidase in peripheral blood mononuclear cells from dengue fever and dengue hemorrhagic fever patients. Southeast Asian J Trop Med HIth 2009; 40 (1): 56-65.
44. Appanna R, Tan LH, Lum CS, Tan PL, Vadivelu J, Devi S. Cross-reactive T cell responses to the non-structural regions of dengue viruses among dengue fever and dengue hemorrhagic fever patients in Malaysia. Clinic Vacc Immunol 2007; 14(8): 969-977.

45. Osmali O, Fong MY, Devi S. A preliminary study of dengue infection in Brunei. Jap J Infect Dis 2007; 4: 205-208.

46. Osmali O, Fong MY, Devi S. Sequence analysis of the E/NS1 gene junction of dengue virus type 2 isolated in Brunei. Southeast Asian J Trop Med HIth 2008; 39 (1): 62-78.

47. Osmali O, Fong MY, Devi S. Complete genome sequence analysis of dengue virus type 2 isolated in Brunei. Virus Research 2008;135(1):48-52..

48. Crill WD, Gwong-Jen LC. Localization and characterization of flavivirus envelope glycoprotein cross-reactive epitopes. $J$ Virol 2004; 78(24) : 13975-13986.

49. Yorgo M, Ogata S, Clement D, Harrison SC. Structure of dengue virus proteins after membrane fusion. Nature 2004; 427:313-319.

50. Kuruvilla JG, Troyer RM, Devi S, Akkina R. Dengue virus infection and immune response in humanized RAG2-/-үc-/- (RAG-hu) mice. Virology 369(1): 143152. 